\title{
Treatment optimization and prognostic considerations for primary squamous cell carcinoma of the thyroid
}

\author{
Wenlong Wang, Qianhui Ouyang, Chaoyang Meng, Lanyu Jing, Xinying Li \\ General Surgery Department, Xiangya Hospital, Central South University, Changsha 410008, China \\ Contributions: (I) Conception and design: W Wang, X Li; (II) Administrative support: X Li; (III) Provision of study materials or patients: W Wang, \\ X Li; (IV) Collection and assembly of data: W Wang, Q Ouyang, X Li; (V) Data analysis and interpretation: W Wang, Q Ouyang, C Meng; (VI) \\ Manuscript writing: All authors; (VII) Final approval of manuscript: All authors. \\ Correspondence to: Xinying Li. General Surgery Department, Xiangya Hospital, Central South University, No. 87 Xiangya Road, Changsha 410008, \\ China. Email: lixinyingcn@126.com.
}

\begin{abstract}
Background: Primary squamous cell carcinoma of the thyroid (PSCCT) is a rare aggressive malignancy that usually presents in an advanced stage and has a poor prognosis. Our study aimed to investigate the clinical characteristics, treatment, and prognosis of PSCCT.

Methods: We retrospectively reviewed the medical information of patients with PSCCT diagnosed from January 2006 to May 2018 at Xiangya Hospital. Survival analysis was conducted using the Kaplan-Meier method, and Log-Rank tests were performed for statistical testing.

Results: We identified 12 patients with PSCCT (nine males and three females), accounting for only $0.19 \%$ of all thyroid cancer diagnosed during this time period. The median age of these patients was 59.5 years old and their symptoms included neck masses $(n=5)$, hoarseness $(n=2)$, dyspnea $(n=1)$, dysphagia $(n=1)$ and neck pain $(n=1)$. Four patients were in stage IVA, five were stage IVB, and three patients were stage IVC. Six patients underwent comprehensive treatment (surgery + radiotherapy or surgery + radiotherapy + chemotherapy) and the remaining patients received radiotherapy and/or chemotherapy. The 6-month survival rate was $66.7 \%$, compared to a 1 -year survival rate of $25.0 \%$, with a median overall survival time was 10.5 months. Kaplan-Meier analysis showed that the comprehensive treatment was superior to radiotherapy and/or chemotherapy $(\mathrm{P}=0.003)$.
\end{abstract}

Conclusions: PSCCT is a rare type of thyroid cancer that is highly invasive and has a poor prognosis. We show that a comprehensive treatment plan can significantly improve patient survival.

Keywords: Squamous cell carcinoma; thyroid; radiotherapy; chemotherapy

Submitted Aug 14, 2019. Accepted for publication Nov 04, 2019.

doi: $10.21037 /$ gs.2019.11.07

View this article at: http://dx.doi.org/10.21037/gs.2019.11.07

\section{Introduction}

Thyroid cancer is the most commonly diagnosed endocrine malignancy and has a rapidly increasing incidence worldwide (1). Primary squamous cell carcinoma of the thyroid (PSCCT) is a rare type of thyroid cancer that accounts for less than $1 \%$ of all cases $(2,3)$. The first case of PSCCT was reported by Vonkarst in 1858 (4). Patients with PSCCT often present at an advanced stage because of the rapidly progressing and highly invasive nature of the malignancy. These PSCCT characteristics also make it difficult to achieve negative margins during radical resection of the tumor. Because of the difficulties of removing PSCCT surgically and its lack of sensitivity to radiotherapy or chemotherapy, patients with PSCCT have an extremely poor prognosis, with median survival times after diagnosis of only 5-8 months $(5,6)$. The clinical behavior of PSCCT resembles anaplastic thyroid carcinoma, another difficult to treat tumor of the thyroid with a poor prognosis $(3,7)$. 
The published literature regarding PSCCT is limited, and includes only a few individual case reports and studies with small sample sizes $(6,8-11)$. Thus, optimal approaches for treating patients have not yet been developed and there are no standardized management guidelines to treat PSCCT. Radiotherapy or chemotherapy is the only option for treating patients with unresectable PSCCT. Yet even for patients receiving surgery, potential benefits of additional treatment afterward are also unknown $(3,12,13)$. To address this, we retrospectively identified patients with PSCCT who received treatment at Xiangya Hospital during 2006-2018. We used the clinical characteristics and outcomes of these patients to develop comprehensive diagnostic and treatment approaches for patients with PSCCT.

\section{Methods}

\section{Patient demographics}

We performed this retrospective study after receiving approval from the Ethics Committee of Xiangya Hospital, Central South University in Changsha, China (No: 201901005). We identified 6,190 cases of thyroid cancer from January 2006 to May 2018, yet only 12 patients (nine males and three females) met the study's inclusion criteria. These cases accounted for $0.19 \%$ of all thyroid cancers at our hospital. The patients ranged in age from 38 to 79 years old, with a median age of 59.5 years old. Nine of the patients had cervical lymph node metastasis and three had lung metastases.

\section{Inclusion criteria}

(I) Rough-needle aspiration biopsy or postoperative histopathology confirming a diagnosis of PSCCT; and (II) complete clinical data.

\section{Exclusion criteria}

(I) Metastatic/secondary squamous cell carcinoma histopathology; or (II) the primary tumor focus was unknown and was initially diagnosed as squamous cell carcinoma of a thyroid adjacent organ (e.g., esophagus, pharynx/throat, lung).

\section{Treatment strategies}

(I) Surgery: the types of surgical resection included radical, extensive, and palliative resection. Radical resection is a total thyroidectomy plus cervical lymph node dissection.
Extended resection removes the primary tumor focus and surrounding invaded tissue. Palliative resection is not intended to be curative and aims for symptomatic improvement through removal of part of the primary focus or metastatic lymph nodes, leaving visible cancerous tissue behind. (II) Radiotherapy: radical local radiotherapy was given at a dose of 60-86 Gy. (III) Chemotherapy: taxol + platinum or anthracycline + platinum. (IV) Comprehensive treatment: surgery + radiotherapy or surgery + radiotherapy + chemotherapy.

\section{Statistical analysis}

Statistical analysis was performed using SPSS19.0. Survival analysis was conducted using the Kaplan-Meier method, and Log-Rank tests were performed for statistical testing. $\mathrm{P}<0.05$ was considered statistically significant.

\section{Results}

\section{Clinical characteristics}

The main clinical symptoms were neck mass $(n=5)$, hoarseness $(n=2)$, dyspnea $(n=1)$, dysphagia $(n=1)$, and neck pain $(n=1)$ yet two patients had no clinical symptoms at all. Histological evaluation of tumor grade revealed two, one, two, two, and five cases of high, high-moderate, moderate, moderate-low, and poor differentiation, respectively. There were five cases $(41.67 \%)$ where the tumor diameter was $>4 \mathrm{~cm}$, with the remaining seven cases (59.33\%) having tumors $\leq 4 \mathrm{~cm}$ in diameter. All patients were staged according to the American Joint Committee on Cancer (AJCC) staging manual for anaplastic thyroid cancer: stage IVa $(n=4)$; stage IVb $(n=5)$; stage IVc $(n=3)$ (Table 1).

\section{Treatment response}

Six patients underwent rough needle biopsy without surgery. The other six patients received thyroidectomy, in which three, two, and one received radical, extensive, and palliative resection, respectively. Cervical lymph node dissection was performed on all six of these patients and four of six (66.7\%) had cervical lymph node metastasis that was confirmed by histopathology. 11 patients underwent radiotherapy (60-86 Gy). Six patients received chemotherapy, including paclitaxel + cisplatin in for cases and docetaxel + cisplatin for the other two cases. The outcomes of these patients included five patients who had a partial response and four 
Table 1 Clinicopathological features and survival

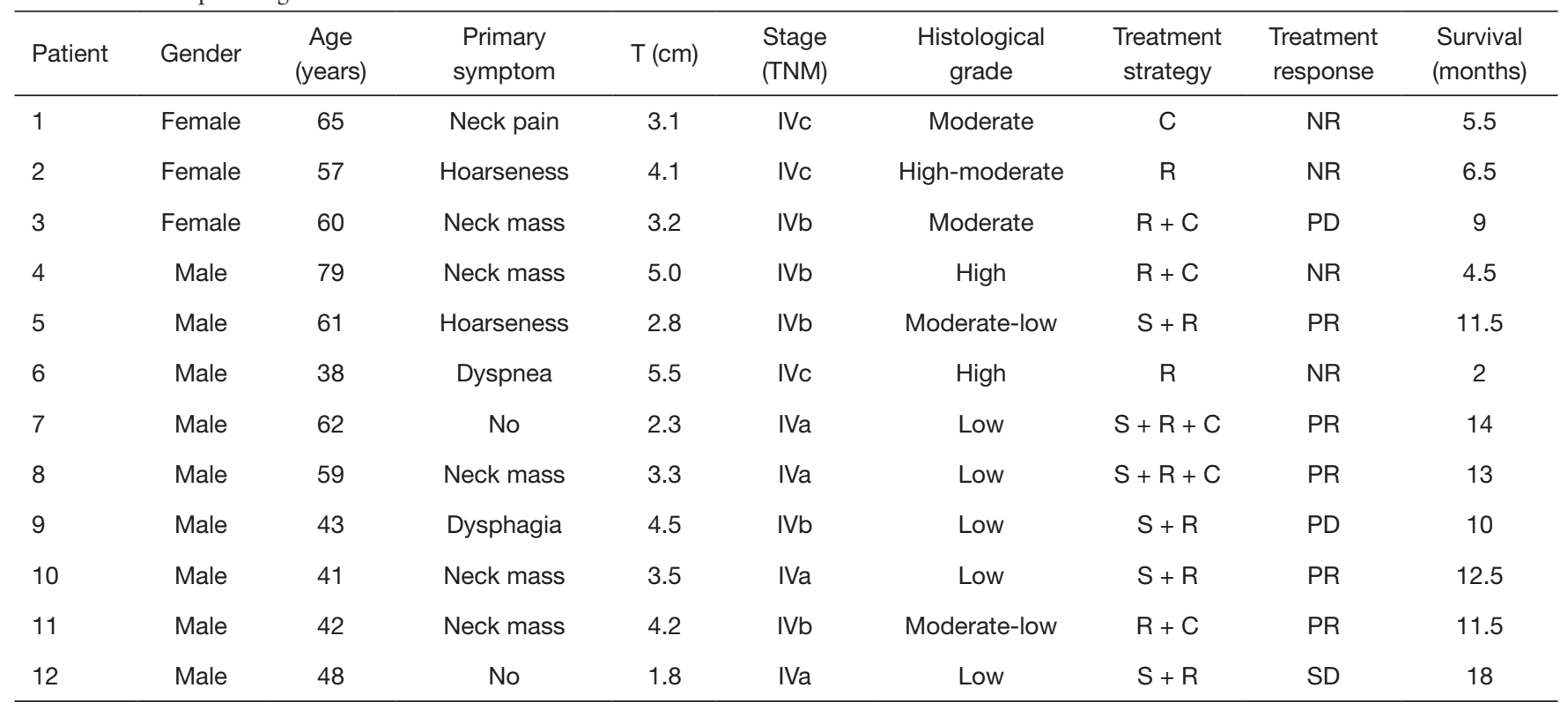

C, chemotherapy; R, radiotherapy; S, surgery; NR, no response; PR, partial response; PD, progressive disease; SD, stable disease.

who did not respond at all. Five of the patients who did respond developed both local and distant recurrence within 11 months Two patients had a progressive disease and one patient who underwent surgery (radical resection)+ radiotherapy (paclitaxel + cisplatin) + chemotherapy (68 Gy) achieved stable disease and survived for 18 months (Table 1).

\section{Prognostic and survival}

The 6-month survival rate for patients with PTSCC was $66.7 \%$, and the 1 -year survival rate was $25.0 \%$. The median overall survival time for all 12 patients was 10.5 months. The median survival time of patients treated with comprehensive treatment versus radiotherapy and/ or chemotherapy treatment was 12.5 and 6.0 months, respectively. During the follow-up period, local tumor recurrence happened in seven patients and distant metastasis occurred in five patients, which included four cases of lung metastasis and one case with bone metastases. The longest survival time was 18 months of a single patient. KaplanMeier analysis showed that the comprehensive treatment was superior to the radiotherapy and/or chemotherapy ( $\mathrm{P}=0.003)$ (Figure 1).

\section{Imaging}

Computed tomography (CT) scans of these patients showed a mass with an irregular shape, poorly defined boundary, calcifications, and necrosis. The growth of the mass invaded surrounding organs, including the esophagus and trachea. Contrast-enhanced CT showed the mass was unevenly enhanced. The signal was mild to moderate in arterial phase and further strengthened in venous phase (Figure 2).

\section{Histopathology}

The diagnosis of all patients was confirmed using histopathology. Tumor specimens showed a palisade arrangement, intercellular bridges, and keratinization with a cancer pearl. Interstitial fibrous tissue hyperplasia was obvious with mononuclear cells and lymphocytic infiltration. All patients' specimens stained positively for p63, while $41.7 \%(5 / 12)$ were $\mathrm{CK} 19^{+}$and the Ki67 proliferation index ranged from $5 \%$ to $50 \%$. All patients' tumors stained negatively for TG, CD5, CK20, CT and TTF-1.

\section{Discussion}

PSCCT is a rare type of thyroid cancer that accounts for less than $1 \%$ of all thyroid cancers $(2,3)$. Squamous epithelial cells are absent in the normal thyroid gland, aside from the minimal cellular remnants of the thyroglossal duct or branchial clefts, left from embryonic development. 
A

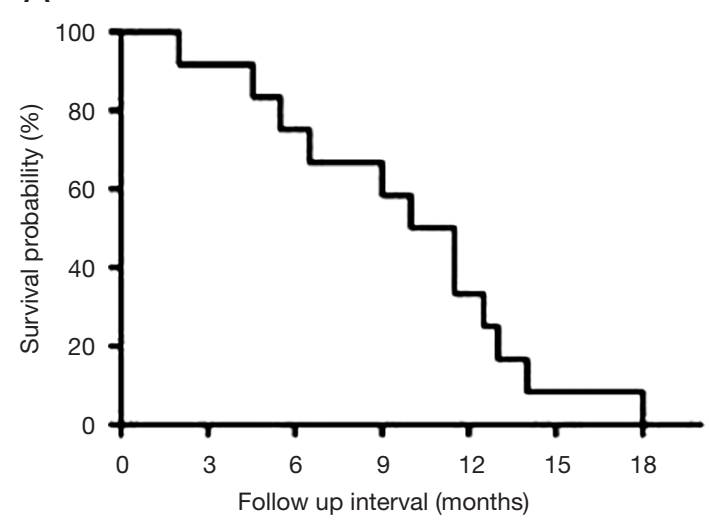

B

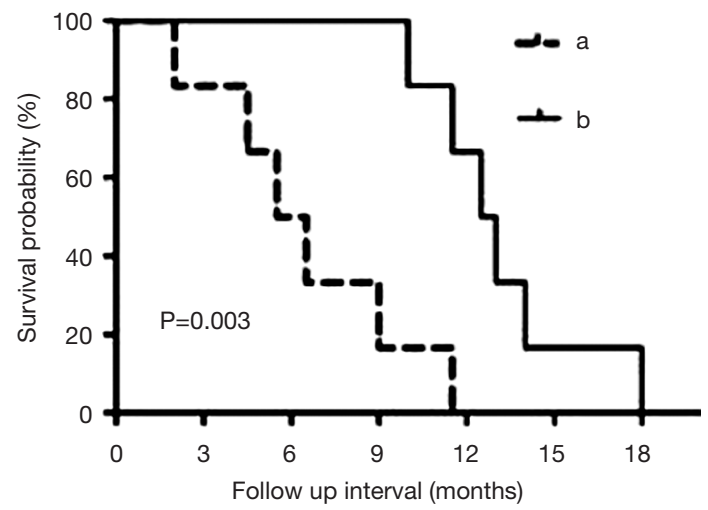

Figure 1 Prognosis and Kaplan-Meier analysis. (A) Kaplan-Meier curve for primary squamous cell carcinoma of the thyroid showing disease specific survival; (B) comparison of survival rate between comprehensive treatment and the radiotherapy and/or chemotherapy where a is radiotherapy and/or chemotherapy $(n=6)$ and $b$ is comprehensive treatment $(n=6)$.
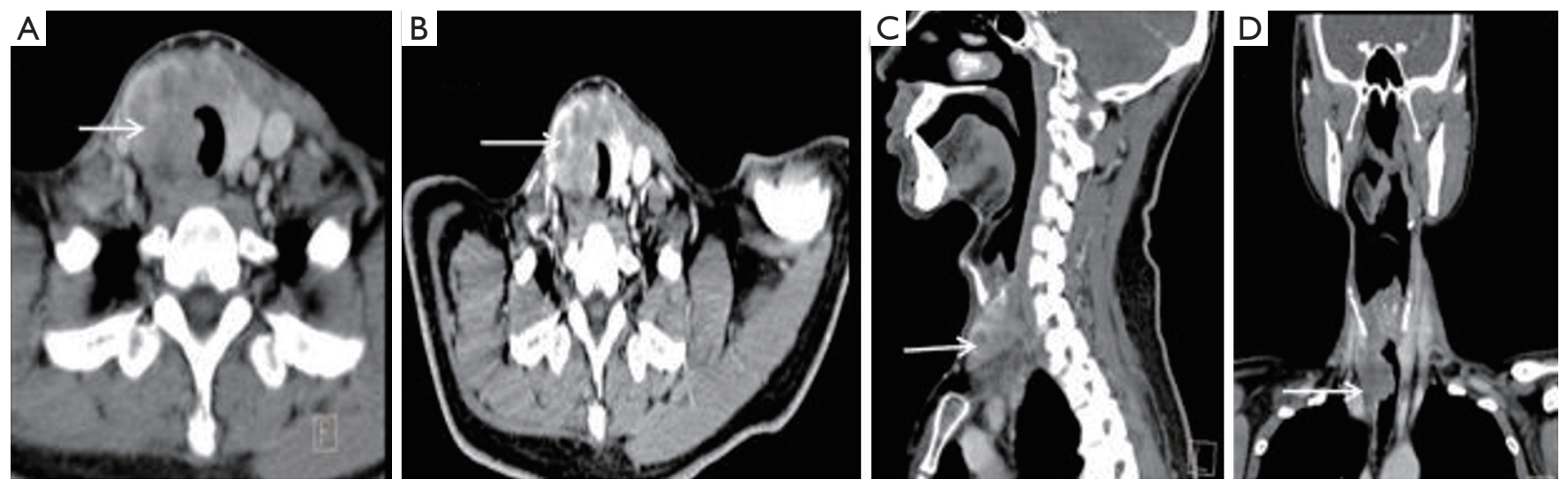

Figure 2 Computed tomography scans of a representative patient with PSCCT. A nodule with mixed density is found in the right thyroid gland, across the isthmus to the left thyroid lobe, with a size of approximately $3.3 \mathrm{~cm} \times 3.4 \mathrm{~cm}$. The right bronchus was compressed, displaced and invaded. Image view: (A,B) horizontal; (C) sagittal; (D) coronal. PSCCT, primary squamous cell carcinoma of the thyroid.

Three theories have been proposed regarding the origin of PSCCT. The embryonic nest theory suggests the squamous cells of PSCCT are derived from embryonic remnants such as thyroid duct, zygomatic arch, posterior corpus callosum, or thymic epithelium (14). The metaphysical theory states that environmental stimuli lead to squamous metaplasia, as seen in squamous thyroid follicular epithelium metaplasia. This theory is supported by observations of focal or extensive squamous metaplasia in pathological specimens from patients with Hashimoto's thyroiditis, nodular goiter, or some other thyroid cancers (15). The de-differentiation theory suggests that existing papillary, follicular, medulla or anaplastic thyroid cancer cells de-differentiate into
PSCCT (16). These competing theories are unified in their belief that squamous metaplasia is a core component of PSCCT etiology (17). When diagnosing PSCCT, it is necessary to exclude tumors that are from adjacent or distant organs (e.g., pharynx, esophagus, lungs, or nasopharynx). Using laryngoscopy, gastroscopy, CT scan, or systemic PET-CT, the possibility of secondary thyroidal squamous cell carcinoma can be ruled out (18-20). Cellular markers such as p63, PAX8, CK7, CK19, CK20, TG, and TTF-1, can also be used to help diagnose PSCCT $(5,8)$. In our study, all patients were diagnosed with PSCCT using histopathology which showed a palisade arrangement, intercellular bridges, and keratinization with a cancer pearl. 

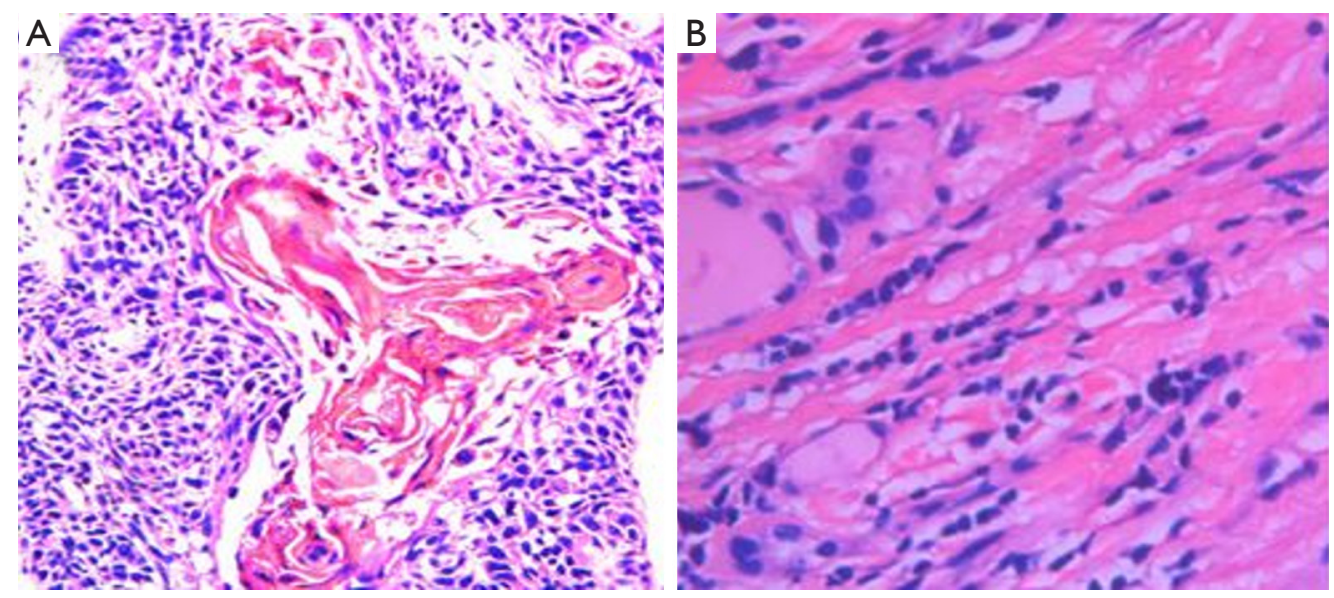

Figure 3 Histopathology using hematoxylin and eosin (H\&E) staining. (A,B) Typical squamous cell carcinoma findings of strands and irregular fields with intercellular bridges, along with a keratinized cancer pearl (40× magnification).

Interstitial fibrous tissue hyperplasia with mononuclear cell and lymphocytic infiltration was also apparent. All patients were $\mathrm{p} 63^{+}$and $41.7 \%(5 / 12)$ also stained CK19 ${ }^{+}$. Ki67 proliferation indices ranged from $5 \%$ to $50 \%$. All patients were $\mathrm{TG}^{-}, \mathrm{CD}^{-}, \mathrm{CK}^{-} 0^{-}, \mathrm{CT}^{-}$, and TTF-1 ${ }^{-}$(Figures 3,4).

Optimal treatment for PSCCT is unknown, as only a few PSCCT cases have been reported and treatment guidelines have not been standardized. PSCCT is characterized by rapid growth and has significant invasive potential for malignancy spread into nearby vessels and the adjacent tissues, including those as small as even in $1-3 \mathrm{~cm}$ in diameter. Some groups believe that it is difficult to stabilize disease progression using only a single treatment strategy $(2,11)$. To date, radical resection combined with postoperative radiotherapy is considered the most effective treatment strategy. In our study, six patients received radiotherapy alone or radiotherapy combined with chemotherapy, which the other patients received comprehensive treatment. The median survival time of these two groups was 6.0 and 12.7 months, respectively. Survival analysis showed that comprehensive treatment was superior to radiotherapy alone or radiotherapy plus chemotherapy. For patients in good condition, chemotherapy can be tried first prior to surgery and radiotherapy. An optimal chemotherapy plan is not yet defined, but it is considered less important compared to surgery and radiotherapy. Struller et al. (9) retrospectively analyzed 50 patients with PSCCT and found that radical resection combined with radiotherapy or chemotherapy led to patients have a longer disease-free survival time. Cook et al. (21) described optimal surgical therapy as hemithyroidectomy or total thyroidectomy, depending on the multifocality, followed by postoperative radiotherapy. The combination of radiotherapy contributes to locoregional control and extends long-term survival time up to 20 months. However, some researchers believe a combination of surgery, radiotherapy, and chemotherapy has little effect on improving prognosis or prolonging survival time in PSCCT patients (19). They theorized that since surgery and radiotherapy are local treatment of cancer lesions, they have little therapeutic effect in cancer cells after blood vessel invasion and distant metastasis happen. They concede that the effect of chemotherapy in this case is still undefined. Although PSCCT is resistant to radiotherapy and has limited response to chemotherapy, we believe that palliative radiotherapy or chemotherapy is a good strategy to reduce tumor load and prolong diseasefree survival time when the tumor is unresectable or radical resection is impossible.

Yasumatsu et al. (3) reported that four patients with PSCCT obtained successful response to oral Lenvatinib, in which one patient achieved partial response, and three showed good response and reached stable status. Lenvatinb is a multi-receptor tyrosine kinase inhibitor that targets a variety of angiogenic and carcinogenic signaling pathways. It has been approved in Japan for the treatment of unresectable thyroid carcinoma, including anaplastic thyroid carcinoma (12). Lenvatinb has already shown considerable promise in the treatment of liver cancer and other types of cancer (22). In addition to surgery, radiotherapy, and 

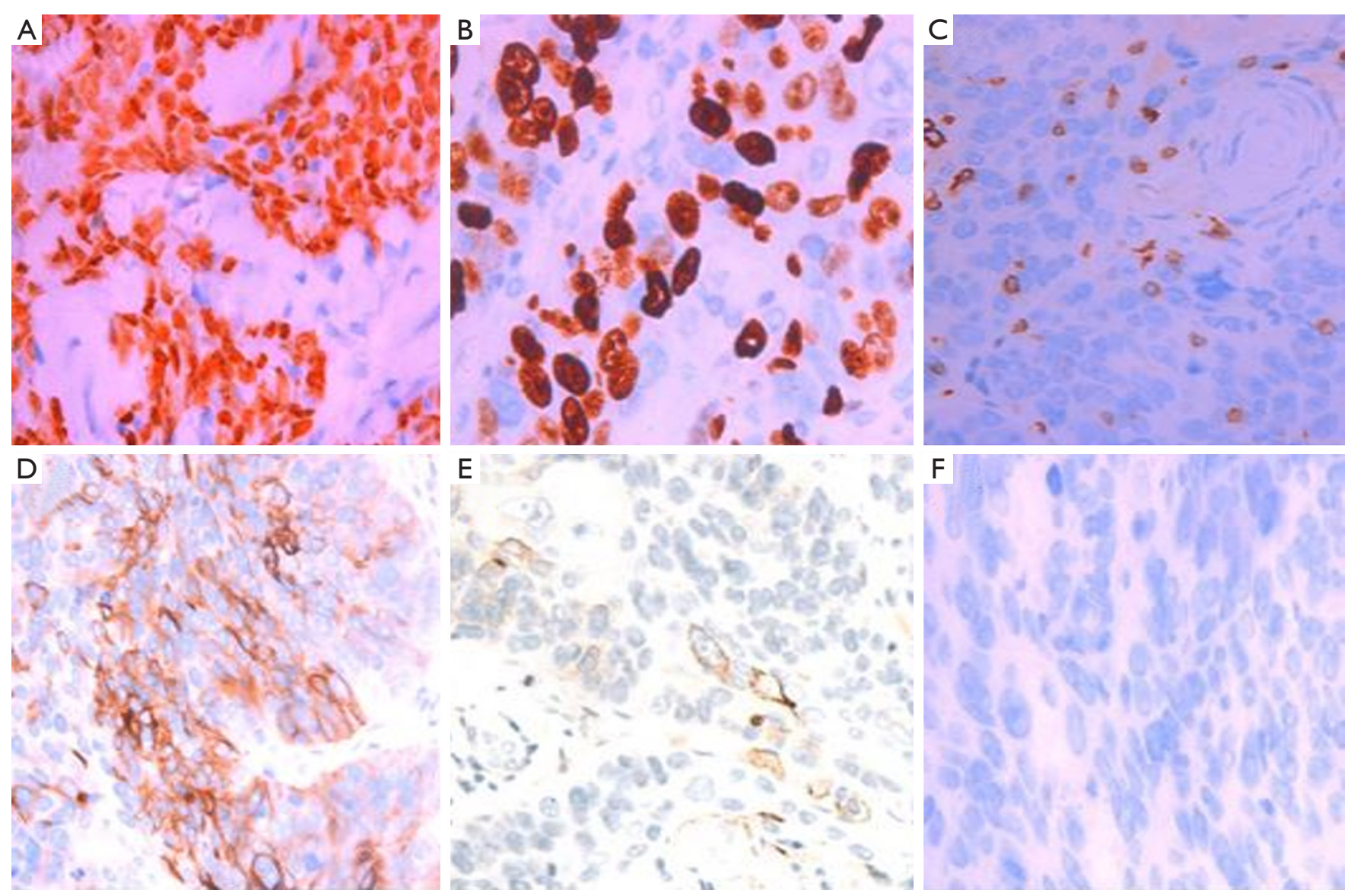

Figure 4 Immunohistochemical (IHC) features of PSCCT (40× magnification). IHC staining for (A) p63, (B) Ki67, (C) CD5, (D) CK19, (E) thyroglobulin, or (F) TTF-1 (4F). p63 and CK19 was deemed positive and thyroglobulin, CD5, and TTF-1 did not stain (negative). Ki67 proliferation index was $50 \%$. PSCCT, primary squamous cell carcinoma of the thyroid.

chemotherapy, small molecule inhibitors such as lenvatinb may provide another treatment option for PSCCT patients, that could contribute to prolonged survival.

PSCCT has an extremely poor prognosis due to its rapid growth rate and highly invasive nature. Most patients die within a year of diagnosis and the median survival time is 5 to 8 months $(3,5)$. The main causes of death are attributed to local recurrence and metastasis. A study using the US cancer database retrieved 242 patients with PSCCT from 1973 to 2012, showing that the 5-year overall survival rate and disease-specific survival rate was $16 \%$ and $21 \%$, respectively. The median survival time was 9.1 months (23). Comparatively, we found in our study that the 6-month survival rate of patients with PSCCT was $66.7 \%$, the 1 -year survival rate was $25.0 \%$, and the median survival time was 10.5 months. An individual participant data meta-analysis showed patients with R0 resection had a 3-year survival rate
(3YSR) of $43.1 \%$ and median survival time of 23 months (4). However, patients receiving R1 resection had a 3YSR of only $15.9 \%$ and a median survival time of 4 months. Thus, it appears that surgical resection with negative margins is an important factor that affects the prognosis of PSCCT. Additionally, markers such as p53 and Ki67 (24) may play a role in predicting the prognosis of PSCCT as positivity for these two markers specifically was an indicator of poor prognosis.

\section{Conclusions}

PSCCT is a rare type of thyroid cancer with highly invasive potential and is associated with a poor prognosis. Comprehensive treatment is conducive to improving patient survival. However, superior surgical approaches in PSCCT have not been clearly defined and radiotherapy 
dosing as well as optimal chemotherapy agents are yet to be elucidated.

\section{Acknowledgments}

We would like to thank our patients who allowed us to make this study possible. We thank MedEditing LLC for its linguistic assistance during the preparation of this manuscript.

Funding: This work was supported by the National Natural Science Foundation of China (grant No. 81672885).

\section{Footnote}

Conflicts of Interest: The authors have no conflicts of interest to declare.

Ethical Statement: The authors are accountable for all aspects of the work in ensuring that questions related to the accuracy or integrity of any part of the work are appropriately investigated and resolved. This study was approved and supervised by the Ethics Committee of Xiangya Hospital (No. 201901005).

\section{References}

1. Siegel RL, Miller KD, Jemal A. Cancer Statistics, 2017. CA Cancer J Clin 2017;67:7-30.

2. Yang S, Li C, Shi X, et al. Primary Squamous Cell Carcinoma in the Thyroid Gland: A Population-Based Analysis Using the SEER Database. World J Surg 2019;43:1249-55.

3. Yasumatsu R, Sato M, Uchi R, et al. The treatment and outcome analysis of primary squamous cell carcinoma of the thyroid. Auris Nasus Larynx 2018;45:553-7.

4. Yang S, Li C, Shi X, et al. Primary Squamous Cell Carcinoma in the Thyroid Gland: A Population-Based Analysis Using the SEER Database. World J Surg 2019;43:1249-55.

5. Yamazaki H, Iwasaki H, Okubo Y, et al. Two cases of thyroid gland invasion by upper mediastinal carcinoma. Endocrinol Diabetes Metab Case Rep 2019. doi: 10.1530/ EDM-19-0028.

6. Koyama S, Fujiwara K, Nosaka K, et al. Immunohistochemical Features of Primary Pure Squamous Cell Carcinoma in the Thyroid: An Autopsy Case. Case Rep Oncol 2018;11:418-24.

7. Basnet A, Pandita A, Fullmer J, et al. Squamous Cell
Carcinoma of the Thyroid as a Result of Anaplastic Transformation from BRAF-Positive Papillary Thyroid Cancer. Case Rep Oncol Med 2017;2017:4276435.

8. Lichiardopol C, Surlin V, Foarfa MC, et al. Primary squamous cell carcinoma of the thyroid: a case report. Rom J Morphol Embryol 2016;57:831-6.

9. Struller F, Senne M, Falch C, et al. Primary squamous cell carcinoma of the thyroid: Case report and systematic review of the literature. Int J Surg Case Rep 2017;37:36-40.

10. Beninato T, Kluijfhout WP, Drake FT, et al. Squamous differentiation in papillary thyroid carcinoma: a rare feature of aggressive disease. J Surg Res 2018;223:39-45.

11. Del Rosario M, Dasanu C, Tsai H, et al. Primary squamous cell carcinoma of the thyroid with complete response to radical radiotherapy and concurrent cisplatinbased chemotherapy. BMJ Case Rep 2017. doi: 10.1136/ bcr-2016-217143.

12. Tahara M, Kiyota N, Yamazaki T, et al. Lenvatinib for Anaplastic Thyroid Cancer. Front Oncol 2017;7:25.

13. De Vos FY, Sewnaik A, de Wilt JH, et al. Combined therapy for thyroid squamous cell carcinoma. Head Neck 2012;34:131-4.

14. Goldberg HM, Harvey P. Squamous-cell cysts of the thyroid with special reference to the aetiology of squamous epithelium in the human thyroid. Br J Surg 1956;43:565-9.

15. Chaudhary RK, Barnes EL, Myers EN. Squamous cell carcinoma arising in Hashimoto's thyroiditis. Head Neck 1994;16:582-5.

16. Kebapci N, Efe B, Kabukcuoglu S, et al. Diffuse sclerosing variant of papillary thyroid carcinoma with primary squamous cell carcinoma. J Endocrinol Invest 2002;25:730-4.

17. Wygoda A, Rutkowski T, Szczesniak-Klusek B, et al. Primary squamous-cell thyroid carcinoma - a successful treatment with five-year follow-up. Endokrynol Pol 2017;68:592-6.

18. Cai L, Chen Y, Huang Z, et al. Primary Squamous Cell Carcinoma of the Thyroid on FDG PET/CT. Clin Nucl Med 2014;39:1014-6.

19. Syed MI, Stewart M, Syed S, et al. Squamous cell carcinoma of the thyroid gland: primary or secondary disease? J Laryngol Otol 2011;125:3-9.

20. Wang W, Xia F, Meng C, et al. Prediction of permanent hypoparathyroidism by parathyroid hormone and serum calcium 24h after thyroidectomy. Am J Otolaryngol 2018;39:746-50.

21. Cook AM, Vini L, Harmer C. Squamous cell carcinoma of the thyroid: outcome of treatment in 16 patients. Eur J 
Surg Oncol 1999;25:606-9.

22. Kim DY. New Systemic Therapies for Advanced Hepatocellular Carcinoma. Korean J Gastroenterol 2019;73:10-5.

23. Au JK, Alonso J, Kuan EC, et al. Primary Squamous Cell Carcinoma of the Thyroid: A Population-Based Analysis.

Cite this article as: Wang W, Ouyang Q, Meng C, Jing L, Li X. Treatment optimization and prognostic considerations for primary squamous cell carcinoma of the thyroid. Gland Surg 2019;8(6):683-690. doi: 10.21037/gs.2019.11.07
Otolaryngol Head Neck Surg 2017;157:25-9.

24. Kleer CG, Giordano TJ, Merino MJ. Squamous cell carcinoma of the thyroid: an aggressive tumor associated with tall cell variant of papillary thyroid carcinoma. Mod Pathol 2000;13:742-6. 